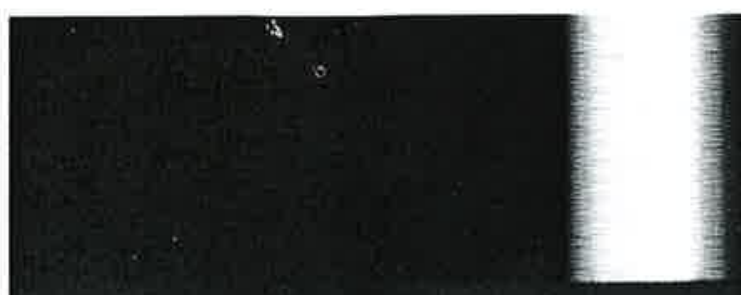

\title{
Teaching mechanical oscillations using an integrated curriculum
}

\author{
M. L. Aiello-Nicosia ${ }^{4}$, E. Balzano ${ }^{3}$, N. Bergomi ${ }^{I}, L . B o r g h i^{5}$, E. Giordano ${ }^{1}$, \\ V. Capocchiani ${ }^{7}, F$. Corni ${ }^{2}, A$. De Ambrosis ${ }^{5}, C$. Marioni ${ }^{I}, P$. Mascheretti ${ }^{5}$, \\ E. Mazzega ${ }^{2}$, M. Michelini ${ }^{7}$, O. Robutti ${ }^{6}, L$. Santi ${ }^{7}, E$. Sassi $^{3}$, R. M. \\ Sperandeo-Mineo ${ }^{4}$, L. Viglietta ${ }^{6}, G$. Vegni $^{1}, P$. Violino $^{6}$ \\ ${ }^{1}$ Dipartimento di Fisica Università di Milano, ${ }^{2}$ Dipartinento di Fisica \\ Università di Modena, ${ }^{3}$ Dipartimento di Fisica Università di Napoli, \\ ${ }^{1}$ Dipartimento di Fisica Università di Palermo, ${ }^{5}$ Dipartimento di Fisica \\ Università di Pazia, ${ }^{6}$ Dipartimento di Fisica Università di Torino, \\ ${ }^{7}$ Dipartimento di Fisica Università di Udine.
}

A set of teaching materials dealing with harmonic oscillations was developed as part of a research project investigating the influence of different pedagogical tools on physics learning. The materials incorporate simulation software and laboratory activities associated with Teacher and Student Units. The project involved research groups at seven Italian universities and the materials have been trialled in high schools nation-wide. This paper describes the experimental protocol for the use of these materials in pilot classes and the cvaluation of student learning and teacher training results. The intervention was found to improve physics education at school praxis level and to promote understanding of the content arca as wel] as appreciation of the role of experiments and simulations in the construction of scientific knowledge.

\section{Introduction}

This paper reports the devclopment and the results of a research project aimed at stimulating interest and meaningful learning by the students through interventions in an emblematic content area of the curriculum, in teacher training process and, directly, in the classroom activities. This involved small-scale curriculum development as well as in-depth classroom research of the teaching-learning processes. Specifically, the project deals with: the preparation of an approach for addressing 'the harmonic oscillator' content area in the framework of current Italian secondary school curricula; the set-up of appropriate teaching strategies related to wellstated educational aims; the structuring of an experimental protocol for the use of the produced materials in pilot classes; and the evaluation of student learning and teacher training results.

The physics content has been chosen since the behaviour of many real systems can be modelled as a composition of hamonic oscillations; therefore, since the hatmonic oscillator is a cornerstone of basic physics learning, to address and over- 
come some common learning difficulties related to this topic can cnhance the study of other topics.

'The project involved research groups at seven Italian universities. It can be classified as 'developmental research' (Lijnse 1995), since it concerns the cyclical process of theoretical reflection, conceptual analysis, small-scale curriculum development, and classroom research on the interaction of the teaching-learning processes.

According to research findings on learning and instruction, in our project the learner is considered as the agent (a) actively constructing his/her view of the natural world, (b) coming to science class with robust models of scientific phenomena and (c) capable of using self-monitoring skills in learning (Eylon and I,inn 1988). These findings are usually taken into account by researchers, but few published papers address how they should be used to improve the curriculum.

Close coupling between educational research and educational practice presents two major problems. The first is methodological: studies in real educational settings are influenced by a number of factors and often the effect of research-based innovations cannot be detected. One of the main factors is the role played by teachers as innovation transformers when they implement curricular innovation in school praxis. The second problem is conceptual: although educational research has substantially advanced in recent years, curricular descriptions are not sufficiently detailed. Research studies generally involve short investigations carried out under rigid control, whereas students in real educational settings are influenced by hard-to-isolate factors and their learning processes last weeks and months.

Methodologically, in order to evaluate the effectiveness of our approach, we compared different groups of students (low, medium and high achievers) looking at such important factors as interest, behaviour and assessment. Since real-world problems of teaching-learning processes tend to be multicausal, multifaceted and non-replicable, we include a qualitative as well as a quantitative approach to data interpretation in order to point out the predictive value of our findings beyond the statistical analysis.

The airm of our project is threefold:

1. 'To improve physics education at school praxis level.

2. To investigate the impact on student learning of a teaching methodology integrating various pedagogical tools and materials.

3. To test the experimentation protocol, that is, the way of conducting the experimentation and its outcome on the actual training of the teachers (their classroom work and the transformations made to the proposed approach).

In the following we describe our research method, qualitative and quantitative results, and finally we draw implications for generalisation of the intervention and for future developments of the research.

The study

This section describes the redesigned curriculum structure and its scenario, the teaching methodology, the pedagogical tools, the participants and the evaluation pattern.

The educe 
n be

lical

evel-

The Mechanical Oscillation Curriculum has been organised with emphasis on the integration of different learning tools (laboratory work, computer simulation, problem-solving) to help student lcarning. The material developed by the research group deals with three topics: Simple Harmonic Oscillator (SHO), Damped Harmonic Oscillator $(D H O)$ and Forced Harmonic Oscillator (FHO). It consists of a Teacher Unit (TU), a Student Unit (SU) with worksheets, and a simulation software, 'Oscillators and Oscillations: O\&O)' (Balzano et al. 1990, Bellomonte et al. 1996).

Two different didactic approaches were suggested for each topic and teachers chose one of them. One Introductory Unit for teachers, 'Many Springs and Many Oscillators', describes different kinds of physical situations that can be modelled by a mass connected to elastic elements.

A key line of the teaching proposal is the focus on a conceptual development path which involves both careful observation of phenomenology and modelling (as mathematical and graphic representations). Each didactic approach is proposed in a defined 'scenario', that is, a set of activitics suggested to students and teacher. A detailed description of the scenario is given in the TU which also includes the objectives of each activity, fairly comprehensive suggestions for experimental work (including some 'how to do it' instructions to build some apparatus) and proposals for homework and software usc.

The software $0 \& O$ is an open learning environment type of interactive graphic simulation program. It combines animation of the oscillator motion and supplies graphs of position and velocity as a function of time as well as the phase space representation of motions. To simulate $\mathrm{SHO}_{\mathrm{s}}, \mathrm{DHOs}$ and $\mathrm{FHOs}$, students are requested to choose the physical parameters of the oscillators and the initial configurations.

In the following, the material developed by the research group is summarised. ${ }^{1}$

Many springs and many oscillators

Three parts can be identified:

1. The first describes oscillators with an oscillating element which is a thin metalic sheet or a metallic wire deformed by bending as well as by twisting.

2. The second describes some physical systems, constituted by springs and masses interacting in various configurations and subjected to different structural constraints.

3. The third shows illustrations and suggestions for constructing the various oscillating systems using simple low-cost materials.

The possibility provided for students to observe functioning models of different devices holds, in our opinion, an important cognitive value since it allows them to recognise that the same physical model is able to describe the behaviours of different systems.

$$
S H O(I)
$$

The educational path suggests the following activities: 


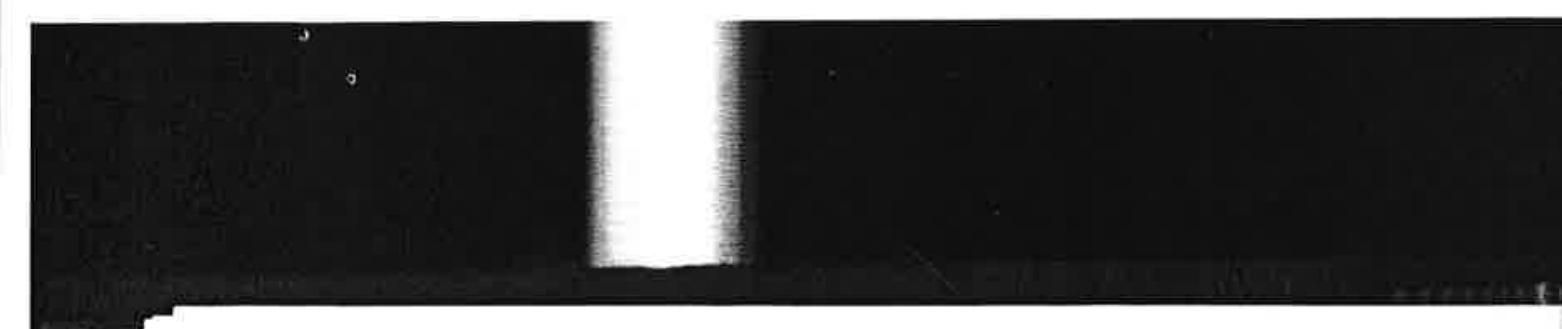

- Observations of periodical phenomena are set up in the classrom in order to identify the relevant variables for their descriptions. The transition from perception to variable definition is made through the phenomenological analysis of a particular periodical motion: the harmonic oscillation of a mass suspended to an helicoidal metallic spring. The measure of the period $z s$. initial amplitude is proposed to help students understand that the period and amplitude of the oscillator are independent quantities.

- Experiments aimed at exploring the elastic force of a spring and measuring its elastic constant $K$ are therefore suggested; measures of the elastic force and oscillation period then follow. A second step proposes experiments to measure how frequency is related to $K$ and the suspended mass. 'The whole set of class data from different small groups of students is therefore analysed and interpreted.

- Frec exploration with $0 \& O$ software is also suggested for comparison with the previously collected experimental data. Graphs of position and velocity as function of time are studied, together with their corrclations. 'I'he meaning and the role of the initial conditions are considered by studying different software simulations; in this way it is possible to address and overcome some common learning difficulties.

- Study of phase space (position-velocity graph) is then proposed to point out the advantages of multirepresentation; the elliptical graphs of harmonic oscillators in this space are studied through software-based activities.

\section{SHO (II)}

Here the starting point is a class discussion about different periodical phenomena and the definition of the physical quantity 'period'. Attention is then focused on the motion of oscillators by means of experimental activities on systems of masses and springs.

The next steps concern the modelling of the observed systems. Students are introduced to simple concepts concerning the numerical integration (Euler method) of the motion equation and are encouraged to carry out a numerical integration using pencil and paper. Then they work with the eomputer simulations of the systems analysed in the laboratory as in SHO (I).

\section{DHO (I)}

In order to link the study of physics to practical applications and thus motivate some students, this unit proposes the study of shock absorbers in a car. A simple model of the car suspensions is presented and the role of shock absorbers emphasized. In laboratory experiments, the behaviour of a damped spring oscillator (made by the students themselves along hints given in SU) is then investigated and compared with the results of computer modelling with different springs and in different damping conditions. An optical technique is proposed for taking measurements, and some suggestions are given to evidence the exponential amplitude decrease. 'The results are then transferred back to the interpretation of the car behaviour, linking technical features of various types of shock absorbers to the underlying physies.

\section{FHO}

'This approach do modelling a partic road containing $\mathrm{re}$ spring sliding on on the sliding ve motion parameter the model using t the amplitue $A(w$ sliding velocity) resonance is qual 
order to ion from ¡ical ana$\mathrm{f}$ a mass eriod $v s$. rriod and

casuring tic force ments to 1e whole analysed

son with locity as meaning different vercome

woint out ic oscil-

nomena used on f. masses

ents are

(Euler umerical ulations

notivate - simple emphascillator stigated $\mathrm{s}$ and in ig meaiplitude the car $s$ to the

\section{$D H O(I I)$}

In order to introduce students to the study of damped oscillators, a set of experiments is outlined, in which a mass is suspended to a spring, the mass being rigidly connected to a spherical body travelling in a fluid (air, water, glycerine). The system exhibits, within good approximation, an exponential decrement of amplitude with time. The immediate tasks for the students are to explore different situations and to measure oscillation period and amplitude versus time. Thus students can begin to understand halving time and pseudoperiod. Further, by changing the diameter of the sphere and the nature (viscosity) of the fluid, they begin to single out the dominant physical parameters. O\&O software, now introduced with the aim of reproducing the observed behaviours, plays an important role allowing a fruitful feedback between thinking and experimental activity.

\section{FHO (I)}

Two experimental set-ups are used to help students recognise that, after an initial time interval where the motion is not regular, the system oscillates with the frequency of the applied force and with an amplitude depending on this frequency. In particular, it is shown that, if friction is low, the amplitude has a maximum when the oscillator eigenfrequency is equal to the frequency of the applied force: this is the resonance condition.

Resonance is then studied by using the computer program $0 \& \mathrm{O}$ which allows students to obtain graphs of the motion of oscillators with the same characteristics observed with the experimental apparatus. Students can change the values of mass, elastic constant, frequency of the applied force, and friction. Specifically, they can explore the dependence of the amplitude $\mathrm{A}(\omega)$ on the frequency of the applied force to find the resonance condition and investigate the influence of the friction on this condition. The passage of the oscillator to the stationary condition can be studied by representing the motion also in graphs of velocity ws. position, reinforcing the meaning of the already proposed (in $S H O(I)$ phase space representation of motion.

\section{FHO (II}

This approach does not give an exhaustive treatment of the topic but it aims at modelling a particular physical phenomenon, i.e. the motion of a car along a wavy road containing regular subsidences. The car is modelled as a mass connected to a spring sliding on a wavy outline that acts as a force whose frequency is depending on the sliding velocity. Students are guided to investigate the variations of the motion parameters of the mass as a function of the sliding velocities by simulating the model using the softwarc O\&O. In particular, they explore the dependence of the amplitue $\mathrm{A}(\omega)$ on the frequency of the applied force (and consequently on the sliding velocity) to find the resonance condition. The influence of friction on resonance is qualitatively investigated. 
The teaching methodology

The proposed classroom activities require the use of different tools organised in a teaching-learning cycle based on different steps: observing, predicting, experimenting (simulating) and feed-back.

In their work students are encouraged to express their predictions before running each experiment/simulation. Making predictions helps students to be engaged in self-monitoring to reconcile their predictions with outcomes. 'The feed-back from numerical or graphic results of their activities is used by students, also through a group discussion, to refine or substantially modify their previous predictions. The results of the different groups are then discussed by the whole class.

Alternating experimental worls and simulations allows students to use results drawn from real word experiences to make predictions about simulated experiments and vice versa. 'This is especially true when students work in groups: predictions made in a co-operative group require discussions in order to evaluate the applicability of past experiences or scientific principles and to justify individual points of view.

The SU requires verbal statements and explanations of the predictions as well as qualitative prediction graphs. Moreover, the proposed activities are designed to encourage students to link different pieces of information by reflecting upon the individual path followed to access and gain new information. 'I'his extra processing can be relegated to home-work and can be a starting point to introduce the next activity.

\section{Evaluation pattern}

The evaluation pattern is divided in three phases:

Phase one: the analysis of the background of the teachers and the students particpating in the experimentation. The descriptor variables are the following: teacher degree and teaching experience, student agc, class history, group-work experience, software literacy, laboratory practice, previously addressed curriculum.

Phase two: a formative evaluation during the experimentation with a twofold objective: to provide information about the 'effective' use of the materials (SUs, experiments and simulations) and to produce feed-back for revision and optimisation of the proposed materials.

Phase three: a sumnative cvaluation carried out at the end of the programme in order to test the student learning and the teacher cvaluation of the materials and of the teaching methodology.

The evaluation instruments have been the following

- two open answer tests aimed at obtaining data on students' knowledge before and after the teaching experiment;

- the group daybook written by each group of students at the end of each class period to summarise the work and to gather evidence about what and how the students learned and what still needs to be clarified;

- a set of interviews given (by the teacher) to small selected groups of students;

- two questionnaries addressed to the teachers, at the beginning and at the cnd of experiment;
- the teacher period (labor tive difficult

Two tests adn the beginning of addition, six stud research group ac

The main thr litative data prov processes in their tions. Since we w us to proceed in evaluations.

Partici

The seven Italiar consequently our nomic levels.

TEACHERS mathenatics or $\mathrm{F}$ according to the interest in innov: with little or no e cular areas.

The teachers town: three befo: classroom worls, meetings were ai experimentation, (laboratory activi were concerned, the TUs. During problems concer mectings were s। outcomes and th apparatus.

STUDENT dents in the class were in the last $y$ in the 17-18 age 
- the teacher diary, a notebook concerning the work carried on each class period (laboratory work, computer work, discussion, lecture, etc.), the operative difficulties and so on.

nised in a 3 experi-

as before ats to be nes. The students, previous he whole

e results l experiıps: preluate the dividual

particteacher rience,

objecxperition of

ime in and of

sefore
Two tests administered to the students were proposed, an Input Test (IT) at the beginning of the experimentation and an Output Test (OT) at its end. In addition, six students out of each class were interviewed by a member of the research group according to a specially designed interview protocol.

The main thrust of the following analysis is a qualitative one; the many qualitative data provided us with a source of rich description and explanation of processes in their local setting, so we could assess local as well as general connections. Since we were using a multisite, multimethod design, this method allowed us to proceed in a relatively formalised way, making substantiated comparative evaluations.

\section{Participant background}

The seven Italian universities participating in the research are nation-wide and consequently our sample of students represents different cultural and socio-cconomic levels.

TEACHERS: 'Twelve teachers have taken part in the project; all are graduated mathematics or physics majors. Six research groups proposed two teachers each according to the following criteria: no less than five years of teaching experience; interest in innovative teaching methods and computer based education coupled with little or no extensive previous experience with experimentation of new curricular areas.

The teachers had, on average, seven meetings with the research group of their town: three before the beginning of the classroom work, one or two during the classroom work, and two or three at the end, after the output test. The initial meetings were aimed at sharing with the teachers the aims and meanings of the experimentation, providing a detailed analysis of the proposed materials (laboratory activities, software and SUs) especially as far as classroom activities were concerned, holding detailed discussion of the teaching strategies proposed in the TUs. During the experimentation, the meetings aimed at solving particular problems concerning the teaching methodology and/or the materials. The final meetings were spent in analysing the evaluation material and in discussing the outcomes and the revision of the SUs, the TUs, the software and the laboratory apparatus.

S'TUDENTS: 'The students involved in the project have been the $285 \mathrm{stu}$ dents in the classes of the participating teachers. Except for onc class the students were in the last ycar of the Iralian science high schools (Liceo Scientifico) and were in the 17-18 age range. 


\section{Results}

The teacher questionnaives

Analysis of the questionnaires gave information concerning the class background as well as the features of previous class work and the evaluation made by the teachers.

All classes before the beginning of the experimentation had studied Kinematics (uniform motion and motion with constant acceleration) and Newtonian Mechanics (the three principles and mechanical energy). They had already used graphs and laboratory aparatus but not computer simulations; only a few classes used software to graph experimental data.

The time spent for the curriculum experimentation was different in the various classes: on average, 13.3 class periods (each class period is 50 minutes) with a standard deviation $\sigma=3 \cdot 0$.

'Teaching practice seems to have been considerably changed during this project. In table I we show data summarising the teacher questionnaires: the time for laboratory and computer work increased with respect to previous practice, while the time spent for lecturing and student assessment diminished. The large values of the standard deviations indicate wide distributions: different classes required different time to obtain the same objectives. The lecture time has been, in all the classes, reduced to a few minutes for the introduction of the topic; after this, the students in small groups (three to four in each group) analysed the corresponding worksheets of the SU in order to plan the laboratory and/or the computer work.

Most of the teachers declared that, before the experimentation, they used the laboratory almost exclusively for demonstration experiments intended for the whole class. During the experimentation all the laboratory experiments and the computer simulations were performed by the students themselves in small group

Table 1. Fraction of class-time for the teaching strategies.

\begin{tabular}{ccc}
\hline & Before & During \\
\hline Lecture and Book Analysis & 0.23 & \\
SU analysis (Group-Work) & $(0.14)^{*}$ & 0.10 \\
Class Discussion & & $(0.06)^{*}$ \\
Laboratory & 0.18 & 0.17 \\
Simulation Software & $(0.09)^{*}$ & $(0.06)^{*}$ \\
Assessment & 0.17 & 0.33 \\
& $(0.06)^{*}$ & $(0.11)^{*}$ \\
Other & 0.14 & 0.39 \\
& $(0.08)^{*}$ & $(0.06)^{*}$ \\
& 0.22 & 0.03 \\
& $(0.08)^{*}$ & $(0.03)^{*}$
\end{tabular}

* Standard deviation. settings: the studes model what they $c$

Test an

The I'T contains abilities to draw, l edge and underst 2 etc.). Splitting the has been verified?

Figure 1 shov sub-tests: the two deviation. Figure students performi

The reliabilit: Fruchter 1973), is geneity of the test correlation coeffic

The OT is $\mathrm{m}$ standing of the $\mathrm{m}$ FHO using the gr test is $r_{t r}=0.87, v$ shows the $\mathrm{O}^{\prime} \mathrm{T}$ di negatively skewee our case, that mor

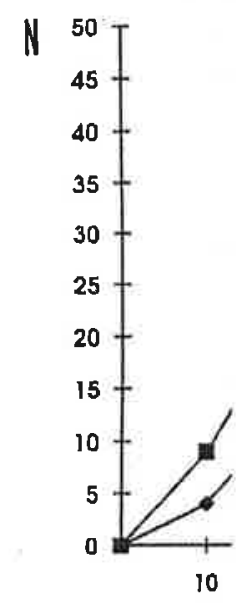

Figure 1. Dist IT-Subtests value of $G$ The mean $\sigma_{\mathrm{CS}}=19.2 \%$ 
settings: the students collected data, analysed them and ran simulations in order to model what they observed in the laboratory.

\section{'Test analysis}

The J'T contains 42 items divided in two sub-tests: the first to ascertain the abilities to draw, understand and interpret graphs; the second to ascertain knowledge and understanding of basic dynamic concepts (velocity, accelcration, force, etc.). Splitting the 42 items into two sub-tests was done by the researchers and it has been verified that the items in each sub-test were significantly correlated.

Figure 1 shows the distributions of percentages of right answers in the two sub-tests: the two curves are bell-shaped with similar average value and standard deviation. Figure 2 shows the sume distribution for the whole test and for the 242 students performing both IT and OT.

The reliability $r_{\mathrm{lt}}$ of the whole test, calculated by the KR 20 (Guilford and Fruchter 1973), is $r_{\mathrm{tt}}=0.84$, which can be considered good given the non-homogeneity of the test. However, the two sub-tests are highly correlated: the Pearson correlation coefficient (Guilford and Fruchter 1973) is $r_{\mathrm{s}}=0.54(\mathrm{p}<0.001)$.

The OT is more homogeneous than the IT: in fact, it ascertains the understanding of the most important concepts concerning the topics $S H O, D H O$ and FHO using the graphic language. The OT contains 23 items. The reliability of the test is $r_{u}=0.87$, which can also be considered good for such a test length. Figure 2 shows the OT distribution of percentages of right answers; the curve shape is negatively skewed: its median is on the right of the mean and this indicates, in our case, that more than $50 \%$ of our sample gave more than $70 \%$ of right answers.

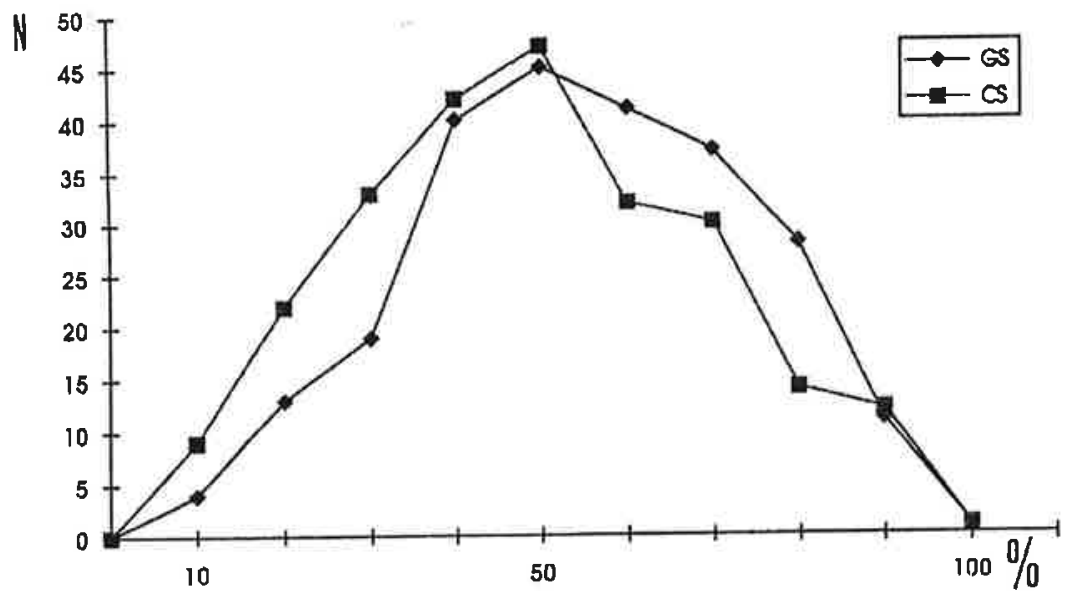

Figure 1. Distributions of the percentage of correct answers to the two IT-Subtests: GS (Graphic Subtest) and CS Concept Subtest). The mean value of $\mathrm{GS}$ is $\mathrm{M}_{\mathrm{GS}}=50.2 \%$ with a standard deviation $\sigma_{\mathrm{GS}}=18.4 \%$ ). The mean value of $\mathrm{CS}$ is $\mathrm{M}_{\mathrm{CS}}=44.1 \%$ with a standard deviation $\sigma_{\mathrm{CS}}=19.2 \%$. 


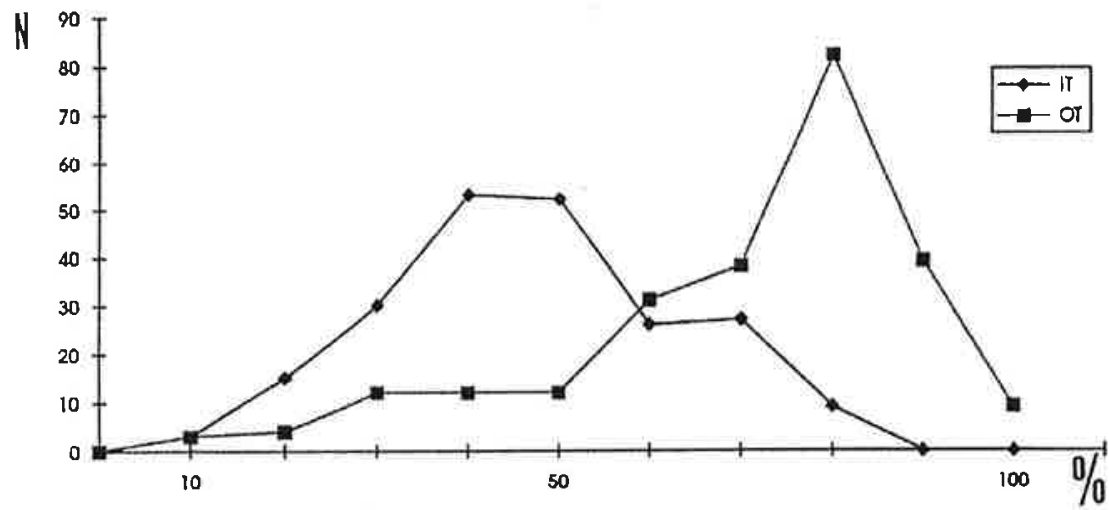

Figure 2. Distributions of the percentage of correct answers of the two total tests I'T (Input Test) and OT (Output Test). The mean value of IT is $\mathrm{M}_{\mathrm{IT}}=47.1 \%$ with a standard deviation $\sigma_{\mathrm{IT}}=16.1 \%$. The mean value of OT is $\mathbf{M}_{\mathrm{OT}^{\mathrm{T}}}=73.2 \%$ with a standard deviation $\sigma_{\mathrm{O}^{\mathrm{T}}}=20.1 \%$.

Since most of our students seem to have achieved the proposed learning objectives, we tried to identify the factors influencing learning in terms of both individual learning and group outcomes. Three groups have been identified on the basis of IT achievement scores: high (H), medium (M) and low (L) achievement level, grouping students whose scores $\mathbf{S}$ were respectively: $\mathbf{S} \geq \mathbf{M}_{1}+\sigma / 2$, $\mathrm{M}_{\mathrm{I}}-\sigma / 2<\mathrm{S}<\mathrm{M}_{\mathrm{I}}+\sigma / 2, \mathrm{~S} \leq \mathrm{M}_{\mathrm{l}}-\sigma / 2$ (with $\mathrm{M}_{\mathrm{I}}=\mathrm{IT}$-mean and $\sigma=\mathrm{I}^{\prime} \mathrm{T}$ standard deviation). Each group contained approximately a third of the total sample $(H=79, M=83$ and $\mathrm{L}=80)$. For each group the means of the IT, of its two sub-tests and of the O'T have been calculated. 'Table 2 shows these results together with the ratios between the output means and the three input means. This analysis clearly shows that the L-group is Low in both the subtests and had the best improvement in the output results.

In order to construct group learning profiles and to look for insights into individual learning outcomes, the $\mathrm{M}$ and the $\mathrm{L}$ groups have been more carefully analysed: the $\mathrm{H}$ group shows almost full use of concepts and abilities and, in our opinion, could proced in constructing its learning almost independently from the proposed teaching methodology. The two groups $M$ and $L$ together inclucied 162 students: $54 \%$ of them show an achievement level improvement in the O'T (i.e., improve from L-level in the IT to $\mathrm{M}$ or H levels in the OT, or from M-level in the IT to H-level in the O'T'), 36\% maintained their positions in the $L$ or M levels and only $10 \%$ made their position worse (i.e., from the M-level in the I'I to the L-level in the $\left.\mathrm{O}^{\prime} \mathrm{T}\right)$.

The performances of the 12 classes have been separately analysed: the range of the percentage of correct answers for I'T is $28 \%-70 \%$ and for O'I is $47 \%-93 \%$. Based on I'T results the twelve classes have been grouped in three groups of four classes: Low, Medium and High type of classes. Table 3 shows IT and OT means and standard deviations for the three class groups as well as ratios between l'l and O'T means. 'T'he significance of difference between group means have been analysed. 'The Student's t-test (Guildford and Fruchter 1973) shows that for IT as
TEACHING MECLIANICAL

Table 2. Fractions

\section{IT'-mean GS-mean CS-mean \\ O'T-mean}

O'T-mean/IT-m $\mathrm{O}^{\prime} \mathrm{T}$-mean/GS-m OT-mean/CS-m

IT'-mean = Mean Fraction CS-mean = Mean Fraction CS-mean $=$ Mean Fraction O' '-mean = Mean Iraction Or $[-$ mean $/ \mathrm{I} T-$ mean $=$ ratio O'I'-mean/GS-mean = ratic $\mathrm{OT}-$ mean/CS-mean = ratic

well as for OT, the Moreover, table 3 shr ones is rather similar trend resulting from ! (Table 2).

Table 3 also sho formance (scale 1-5).

Table 3.

TES'T'EVALUA IT-mean

O'l'-mean

O'T-mean/IT -

TEACHER-EVAI Input-Lva

Output-Ew

Output/InT

IT - mean $=$ Mean Fracti OT-mean $=$ Mean Fracti "The figures in brackets

Input-Eval. $=$ Mean Init Output-Eval. $=$ Mean $\mathrm{Fi}$

-The figures in brackets 


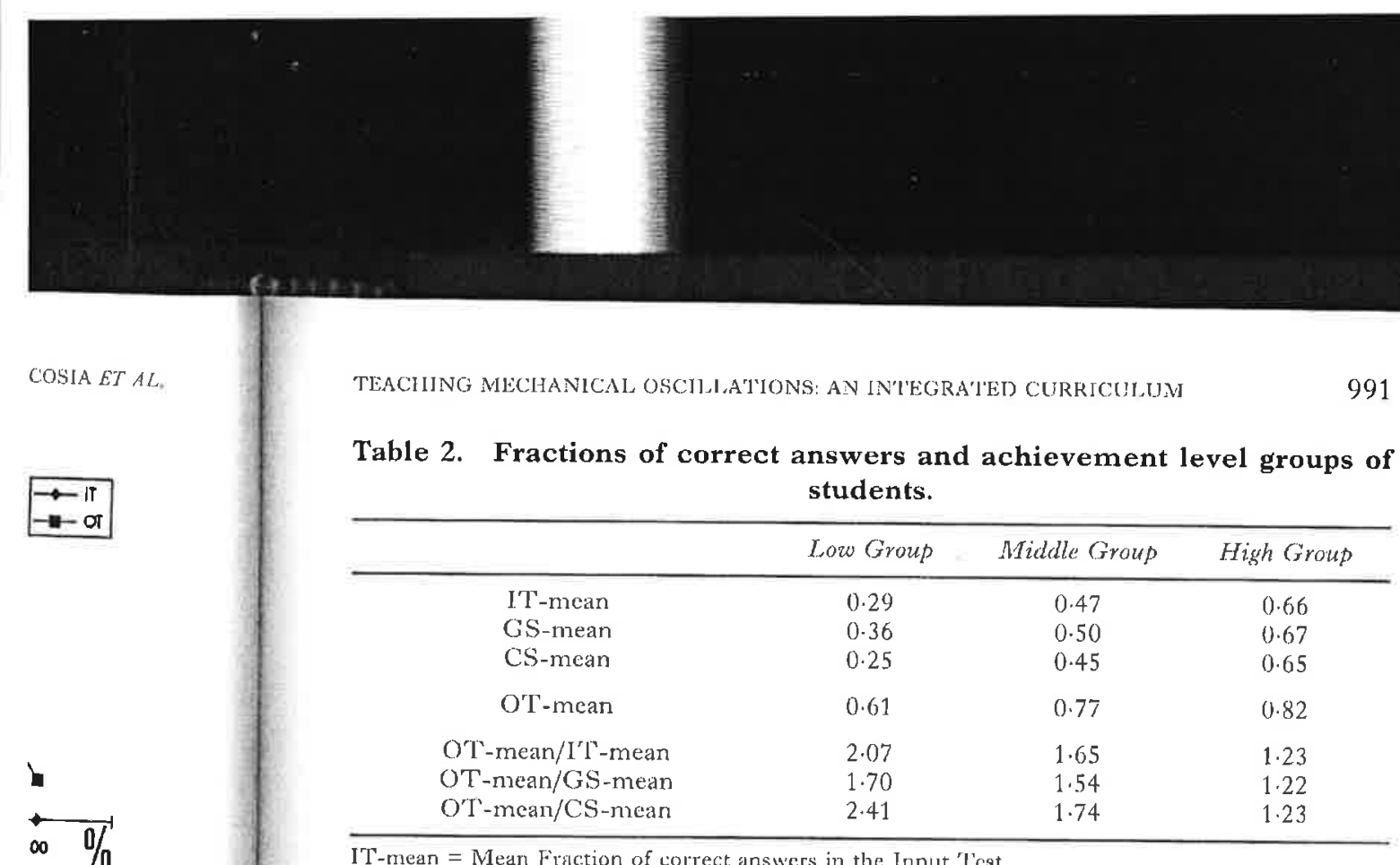

IT-mean = Mean Finction of correct answers in the Input 'I'est

GS-mean = Mean Fraction of correct answers in the Graphic Subtest of IT

CS-mean = Mean Fraction of corfect answers in the Concept Subtest of IT

$\mathrm{O}^{\prime} \mathrm{l}^{\prime}$-mean $=$ Mean Fraction of correct answers in the Output T'est

$O^{\prime} \mathrm{T}^{\prime}$-mean/ $\mathrm{I}^{\prime} \mathrm{l}$-mean = ratio between $\mathrm{O}^{\mathrm{T}} \mathrm{T}$ and $\mathrm{I}^{\prime} \mathrm{T}$ means

$\mathrm{OT}^{-}-\mathrm{mean} / \mathrm{GS}-\mathrm{mean}=$ ratio between $\mathrm{O}^{\prime} \mathrm{T}$ and $\mathrm{GS}$ means

$\mathrm{O}^{\top} \mathrm{T}^{\prime}-$ mean/CS-mean $=$ ratio between $\mathrm{OT}^{\mathrm{T}}$ and $\mathrm{CS}$ means

well als for OT, the group means are not significantly different ( $p>0.05)$. Moreover, table 3 shows that the improvement in $\mathrm{O}^{\prime} \mathrm{I}$ results with respect to $\mathrm{I}^{\prime} \mathrm{T}$ ones is rather similar for the three class groups; this result is different from the trend resulting from grouping of students according to their individual I'T results (Table 2).

'l'able 3 also shows the means of the teacher evaluations of the student performance (scale 1-5). 'I This evaluation has been given by the teachers before as well

Table 3. Evaluation of the three groups of classes.

\begin{tabular}{|c|c|c|c|}
\hline & Low Classes & Middle Classes & High Classes \\
\hline \multicolumn{4}{|l|}{ TEST-EVALUAJION } \\
\hline \multirow[t]{2}{*}{ IT'-meaın } & 0.34 & 0.45 & 0.58 \\
\hline & $(0.09)^{*}$ & $(0.12)^{\infty}$ & $(0.13)^{*}$ \\
\hline \multirow[t]{2}{*}{ OT-mean } & 0.57 & 0.68 & 0.85 \\
\hline & $(0.18)^{-1}$ & $(0 \cdot 16)^{*}$ & $(0 \cdot 10)^{*}$ \\
\hline $\mathrm{OT}$-mean/IT-nean & 1.67 & $1 \cdot 5 i$ & 1.47 \\
\hline \multicolumn{4}{|c|}{ 'TEACHER-EVALUA'ION } \\
\hline \multirow[t]{2}{*}{ Input-Ival. } & 2.9 & 2.7 & $3 \cdot 2$ \\
\hline & $(1 \cdot 2)^{*}$ & $(1 \cdot 1)^{*}$ & $(1.4)^{4}$ \\
\hline \multirow[t]{2}{*}{ Output-[ival. } & $3 \cdot 3$ & 2.9 & $3 \cdot 5$ \\
\hline & $(1.5)^{*}$ & $(1 \cdot 3)^{*}$ & $(1 \cdot 6)^{*}$ \\
\hline Output/Input & $1 \cdot 12$ & 1.05 & $1 \cdot 10$ \\
\hline
\end{tabular}

IT-mean = Mean liacrion of correct answers in the Input 'I'est

OT-mean = Mean Fraction of correct answer in the the Ouput 'Test

"The figures in brackets represent the standard deviations

Input-Eval, = Mean Initial Teacher Vahuation (scale 1-5)

Output-Lival, = Mean Final T'eacher Valuation (scale 1-5)

*'J he figures in brackets represent the standard deviations 
as after expcrimentation. 'The class mean values are not widely spread: they range from 2.5 to 3.5 for the input evaluation and from 2.5 to 4 for the output evaluation. Table 3 reports the means of the input and the output teacher evaluations for the three groups previously described. The ratios between the two evaluations are reported and the significance of differences between group means has been analysed. In all the cases (for the input evaluation as well as for the output evaluation) the group means result not significantly different $(p>0.05)$.

The analysis of the student interview protocols give indication about individual learning profile and the factors influencing them.

\section{Interview analysis}

Six students from cach class have been interviewed, two from each of the achievement levels (high, middle and low) as assessed by their class teachers.

'The first question asked for the most important thing they thought they learned during the whole class work. The answers evidenced that the students judged as 'the most importart thing': gaining familiarity with the computer, increasing mastery of the graphic language, understanding some phenomena, knowing how to describe them, and working experimentally 'doing things' Students werc aware that they had improved their abilities to observe phenomena, to describe them and to correlate them to everyday events and things. Many pointed out the methodological utility of both laboratory and computer work ('things made using the experiments are more clear, those made using the computer more accurate'). A strong analogy is recognised between the behaviour of the reality and that of its representation: 'if a quantity increases varying a parameter in laboratory, it also increases in its computer simulation'.

A second question concened what the students thought they loarned through the laboratory work, the computer work, the group discussions and the homework. They assessed each experience as important but assigned different aims to each; the laboratory is important to define the problem, to analyse, and to begin a research for a suitable solution autonomously; the computer work is thought uscful to define, to clarify, to complete, to quantify and to verify. A student underlines: "The laboratory work helps me to understand, the computer to make clear and to explain'. Another says: 'The computer helps me to understand the observed things'. The group work is considered useful in order to compare one's ideas with those of the fellow-students and to be able to correct errors through the analysis of similarities and differences. Learning through peer analysis of problems was a highly appreciated strategy, among the most appreciated ones. Homework has been considered useful both to rethink the approaches followed in class activities and to exploit their suggestions in other new situations.

The third question was intended to investigate the students' preferences itmong the different activities. Most of them declared they prefer the laboratory work since it allows a direct relationship with the reality and this implies a greater involvement. However, many recognised a great usefulness to the computer work since 'it allows us to see things that are difficult to see in laboratory', Students have appreciated in $\mathrm{O} \& \mathrm{O}$ software the combination of motion animation and kinematics graph representations. They seemed to need a concrete visualisation of motion besides more abstract representations.
Other question: ing and usefulness , high school textboo graphic representat: to the other ones an more explicit.

Students were class in order to $i$ oscillators. The an: gained a good und instruments not ea ments: the laborato tions 'to imagine it these two aspects $\mathrm{c}$ to describe in deta

Conclu

To evaluate how tl discuss the third o teachers' mode of and the modificati teacher questionn conduct of the cla strategies. As a cr varied appreciably the performance time), but mainly (more time has be sion). Teaching $n$ adapted to the cla pedagogical aspec experiments and/ or class discussion

In order to ev by the tcaching $r$ results as well as

Concerning 1 more detectable teacher input evi evaluation is usu and understandis sample, factors $\mathrm{e}$ the bell-shaped $c$ more than $44 \%$, abilities to use al sidered requiren of curriculum. 
d: they range it evaluation. itions for the aluations are as been anat evaluation)

bout indivi-

he achieve-

ought they le students computer, henomena, ig things'. henomena, ngs. Many uter work y the comiour of the rameter in

:d through omework.

is to cach; o begin a ght useful nderlines: zar and to observed ce's ideas ough the problems omework ass activ-
Other questions concerned particular aspects of SUs, for instance, the meaning and usefulness of the phase space diagram that usually is not presented in the high school textbooks. Students did not find this more difficult than other kinds of graphic representations of motion; rather, they found this diagram complementary to the other ones and useful to analyse what happens when time dependence is not more explicit.

Students were also asked to rephrase the activity paths actually followed in class in order to illustrate and clarify the behaviours of the different kinds of oscillators. The answers showed that most of the students realised that they had gained a good understanding of a complex topic using conceptual and concrete instruments not easy to handle. They seem to have corrcctly used such instruments: the laboratory to 'look more deeply into the reality'. 'The computer simulations 'to imagine its possible behaviours' and to see that the comparison betwcen these two aspects could enhance understanding. Almost all students have been able to describe in detail the activities developed in class and their objectives.

\section{Conclusion}

To evaluate how the aims of our research project have been accomplished, we first discuss the third objective concerning the test of the experimental protocol, i.e. the teachers' mode of conducting the experimentation, that is, their classroom work and the modifications made to the proposed teaching approach. The analysis of the teacher questionnaires and daybooks has shown many differences both in the conduct of the class work and in the way of using the various proposed teaching strategies. As a consequence, the time spent in the curriculum experimentation varicd appreciably in the different classes. This difference cannot be ascribed to the performance of the various classes (Low classes did not always spend more time), but mainly to the role assigned by the teachers to the different strategies (more time has been spent by classes preferring group work and/or class discussion). Teaching matcrials have been used differently: in school praxis they were adapted to the class reality through teacher ideas on both the content and on the pedagogical aspects of the proposal. This implied different ways of carrying out experiments and/or setting up models as well as arranging the working-group and/ or class discussion.

In order to evaluate the second aim, concerning the impact on student learning by the teaching methodologies used in this project, we looked mainly to the test results as well as to the teacher evaluations of the students.

Concerning the input evaluation of different classes, the IT results show a more detectable difference among the various classes than that evidenced by the teacher input evaluation (table 3). 'This can be ascribed to the fact that teacher evaluation is usually an intcgrated process very often unable to isolate knowledge and understanding from other complex abilities. Given the low number of our sample, factors explaining the observed difference cannot be identified. However, the bell-shaped distributions of the IT scores indicate that $50 \%$ of students knew more than $44 \%$ of the required basic dynamic concepts and to have $50 \%$ of the abilities to use and interpret graphics. These knowledges and abilities were considered requirements for the mastery of the content and abilities of the new piece of curriculum. 
'The O'T results also show that low-achieving students have learned new concepts and strengthened their abilities connected with graphics interpretation and use: among them more than $50 \%$ mastered more than $70 \%$ of the content and abilities required. The students with low IT achievement level (Low group in table 2) appear to have gained more by the new teaching strategies, in fact their percentage of correct answers is doubled in OT when compared with $\mathrm{I}^{r} \mathrm{~T}$. A good gain can also be ascribed to the students having medium I'I achievement level (Medium group in table 2), whereas the High group students maintain their initial high mastery.

The analysis of teacher and student group daybooks gives us information about the teaching strategies that could account for the learning gain of Low and Medium student groups. Two factors have been identified: the predictionmaking requirement for all laboratory and computer groupwork and the peer interaction in the context of the co-operative goals of learning process within groups. Prediction-making was one of the most successful aspects of the laboratory and computer activities as proposed in the SUs. Teachers state that student discussion and argumentation set up during this phase of any experiment and computer work were always lively, in walking around the class, teachers report hearing almost every student discussing physics. In writing a prediction, the students had to justify the results they expected to see and, in providing their justifications, they had to evaluate the applicability of either past experience or scientific principles. Very often, prediction slzetches were requested and this implied constructing a group opinion through confrontation of individual ideas within the small group.

Most of the teachers refer to a very satisfying level of involvement of all small group members during laboratory and computer work: most of the students appeared to be actively engaged with peers or computers rather than passively attending the activities of others. However, consideration of the patterns of involvement needs to address the nature of studenl-student and student-computer interaction; this analysis is beyond the objectives of the prescnt research.

The described factors have also been evidenced as effectively affecting student learning in other researches (Lewis et al. 1993, Hennessy et al. 1995, Pozzi et al. 1992, Linn and Songer 1991). A very accurate research project (Kempa and Ayob 1995) (addressing the qucstion of how effective group work in science is in promoting 'learning from others' in group settings) indicates that a significant amount of such learning occurs; the authors cvaluate that about $40 \%$ of information included in students' independent written answers are drawn from knowledge and insights that had initially been contributed to the group discussions by other students.

In conclusion, this project shows that, in the emblematic content area of mechanical oscillations, physies cducation at school praxis level can be improved by using a curriculum which integrates experiments with computer simulations. As reported, we have found relevant evidence of student understanding of the harmonic oscillator model as well as of the role of the experiments and simulations in the construction of scientific knowledge. The most impressive eviclence is drawn from student interviews and from their group daybooks.

While all the causes for this understanding are not clearly evidenced, the proposed approaches appeared to be particularly effective with those students who are usually labelled as low achievers. This aspect of our findings is particularly interesting and will be investigated in the near future through the use of the same teaching stratı mental setting.

\section{Acknou}

We wish to thank operation made this from the Ministr] (MURST).

\section{Note}

1. Teacher and St by the SIF (Societ

\section{Refere}

Batzano, E., Certiar ed Oscillazioni,

BELI.OMON'E, L., BA (1996). Oscilla Fisica).

EYIoN, B. and LIN research persp 251-301.

GULAORI, J, P, ar Education $(\mathrm{Ne}$

HeNnEssy, S., TWI Drapler, S, H vention using : of Scienze Edh

KEMPA, R, F., and Joumal of Scie

LFwIS, E. L., STERN introductory $t$

LIJNSE, P. L. (1995) structurc' of s

LINN, M. C. and Sc dents: what : Teaching, 28 (

Pozzı, S., Hovi J:s, boration on ti 223-29 
